\title{
Phenomenological Classification of Turbulence States in Linear Magnetized Plasma PANTA
}

\author{
Tatsuya KOBAYASHI ${ }^{1)}$, Sigeru INAGAKI ${ }^{2,3)}$, Makoto SASAKI ${ }^{2,3)}$, Yusuke KOSUGA ${ }^{2,3,4)}$, \\ Hiroyuki ARAKAWA $^{5)}$, Fumiyoshi KIN ${ }^{6)}$, Takuma YAMADA ${ }^{3,7)}$, Yoshihiko NAGASHIMA ${ }^{2,3)}$, \\ Naohiro KASUYA ${ }^{2,3)}$, Akihide FUJISAWA ${ }^{2,3)}$, Sanae-I. ITOH ${ }^{2,3)}$ and Kimitaka ITOH $^{1,3)}$ \\ ${ }^{1)}$ National Institute for Fusion Science, National Institutes of Natural Sciences, Toki 509-5292, Japan \\ ${ }^{2)}$ Research Institute for Applied Mechanics, Kyushu University, Kasuga 816-8580, Japan \\ ${ }^{3)}$ Research Center for Plasma Turbulence, Kyushu University, Kasuga 816-8580, Japan \\ 4) Institute for Advanced Study, Kyushu University, Fukuoka 812-8581, Japan \\ ${ }^{5)}$ Teikyo University, Omuta 836-8505, Japan \\ 6) Interdisciplinary Graduate School of Engineering Sciences, Kyushu University, Kasuga 816-8580, Japan \\ ${ }^{7)}$ Faculty of Arts and Science, Kyushu University, Fukuoka 819-0395, Japan
}

(Received 11 March 2017 / Accepted 25 March 2017)

\begin{abstract}
Changing the experimental conditions such as the neutral pressure and the magnetic field strength provides a drastic change in the turbulence states in linear magnetized helicon plasmas. In order to define the turbulence states and their occurrence region a throughout parameter scan in the two-dimensional parameter space was performed. The classification of the turbulence states is carried out phenomenologically based on the turbulence spectrum and the waveform.
\end{abstract}

(C) 2017 The Japan Society of Plasma Science and Nuclear Fusion Research

Keywords: turbulence, linear magnetized plasma, drift wave, structure formation, nonlinear coupling

DOI: $10.1585 /$ pfr. 12.1401019

\section{Introduction}

Magnetically confined plasma is often regarded to be a typical nonlinear medium. The plasma confined in far non-equilibrium state is subject to a variety of instabilities, via which the mean gradient retained by the external heating releases a free energy. Furthermore, there are a lot of possible nonlinear interaction channels among fluctuating variables, since the system is governed both by the electromagnetic dynamics and by the fluid dynamics. Nonlinear evolution of the fluctuations makes the prediction of the plasma dynamics difficult, thus the laboratory plasma is controlled sometimes relying on the operators' experience. The nonlinearly governed turbulence is also known to be the source of the anomalous transport that is one of the most serious obstacles for realizing the thermonuclear fusion reactor. Although no ultimate way has been ever established for clarifying the nonlinear system, parameter scan experiments have succeeded to describe the nonlinear nature of the confined plasma. For example, the H-mode power threshold study is often performed by scanning the line averaged density and the input heating power [1-3].

Concentrated study for the nonlinear turbulence states can be conducted in small laboratory plasma devices (see examples in Refs. $[4,5]$.). In particular, a set of experiments using linear devices such as the Large Mirror Device (LMD), the Large Mirror Device-Upgrade (LMD-
U), and the Plasma Assembly for Nonlinear Turbulence Study (PANTA) has exhibited various nonlinear turbulence states. Most essential two among them are the streamer state and the solitary wave state.

The streamer is characterized by a structure radially stretched and azimuthally localized, as reported in Refs. [6-8]. The existence of the streamers in magnetically confined plasmas is foreseen to reduce the plasma performance, since the streamers act as a radially elongated large convective cell. Clues of the streamers were seen in the torus experiments [9] and in the torus simulations [10]. The streamer structure was initially predicted by an analytical study that exhibited importance of the nonlinear coupling between drift waves and a nonlinearly excited global mode the so-called mediator [11]. Dedicated studies for analyzing the nonlinear coupling in density fluctuations demonstrated the reliability of the predicted model [6-8, 12]. Moreover, a selection rule between the streamers and the zonal flows was proposed in numerical simulation study, showing the importance of the ionneutral collisional damping of drift waves [7,8]. Dynamic coupling between the streamer structure and the parallel flow shear driven Kelvin-Helmholtz type instability, which we call the D'Angelo mode, was also unveiled very recently $[13,14]$.

The solitary wave is characterized by a sawtooth-like waveform in the real space and by a significant funda- 
mental mode and higher order harmonics in the frequency and wavenumber spaces [15]. A dynamic oscillation of nonlinear coupling strength was observed by a local measurement $[16,17]$, which is considered to be a clue to explain the excitation mechanism of the higher harmonics. Depending on the relative phase of the higher harmonics with respect to the fundamental mode, steepening of azimuthal gradient, sometimes called shock front, can occur. Formation mechanism of the shock front is discussed recently [18]. Dynamic competition between the solitary wave and the zonal flow type oscillation was also studied $[15,19,20]$. An abrupt and spontaneous transition between the solitary wave state and the streamer state was also observed [21,22].

Each state can be realized in a parameter window defined in the magnetic field strength $B$ and the neutral pressure $p_{\mathrm{n}}$ in PANTA. Although fundamental nonlinear characteristics of each state were deeply studied, comprehensive understanding of the selection rule between the streamer state and the solitary wave state is still challenging. There is an empirical parameter boundary in the $B-p_{\mathrm{n}}$ space, however, any explicit identification of the parameter boundary has not yet been performed. In this paper, we define the strength of the streamer and the solitary wave phenomenologically, by which the occurrence region of these two states are determined. The article is organized as follows: In Sec. 2, experimental setup is briefly described. In Sec. 3, fundamental spatiotemporal and spectral characteristics of these two states are explained, above which the strength of the streamer and the solitary wave are defined in Secs. 4 and 5, respectively. In Sec. 6 the occurrence regions of the two states are discussed, after which the paper is summarized.

\section{Experimental Setup}

The parameter scan experiments were conducted in a linear magnetized plasma PANTA [23, 24]. As shown in Fig. 1 (a), PANTA has a cylindrical vacuum chamber with a length of $l=4050 \mathrm{~mm}$ and a diameter of $D=450 \mathrm{~mm}$. A homogeneous axial magnetic field in the range of $B=$ $0.04-0.15 \mathrm{~T}$ parallel to the cylindrical axis is produced by a Helmholtz coil system. The magnetic field strength is calculated by the Biot-Savart law with the provided current and the geometry of the coil system. The helicon source with a length of $400 \mathrm{~mm}$ and a diameter of $100 \mathrm{~mm}$ is equipped at one side of the device, inside which the $\mathrm{Ar}$ neutral gas is fed. The neutral pressure is diagnosed with an ionization gauge. The plasma is produced with a double loop antenna with $3 \mathrm{~kW}$ and $7 \mathrm{MHz}$ radio frequency discharge [25]. The typical cylindrical plasma radius is $a \sim 5 \mathrm{~cm}$. A stainless end-plate at the other side of the device terminates the plasma column. Two baffle plates at both sides of the chamber having an inner diameter of $15 \mathrm{~cm}$ maintain the neutral pressure constant in the confinement region [26]. The typical operation conditions are summarized in Table 1.

The plasma turbulence is mainly diagnosed with two
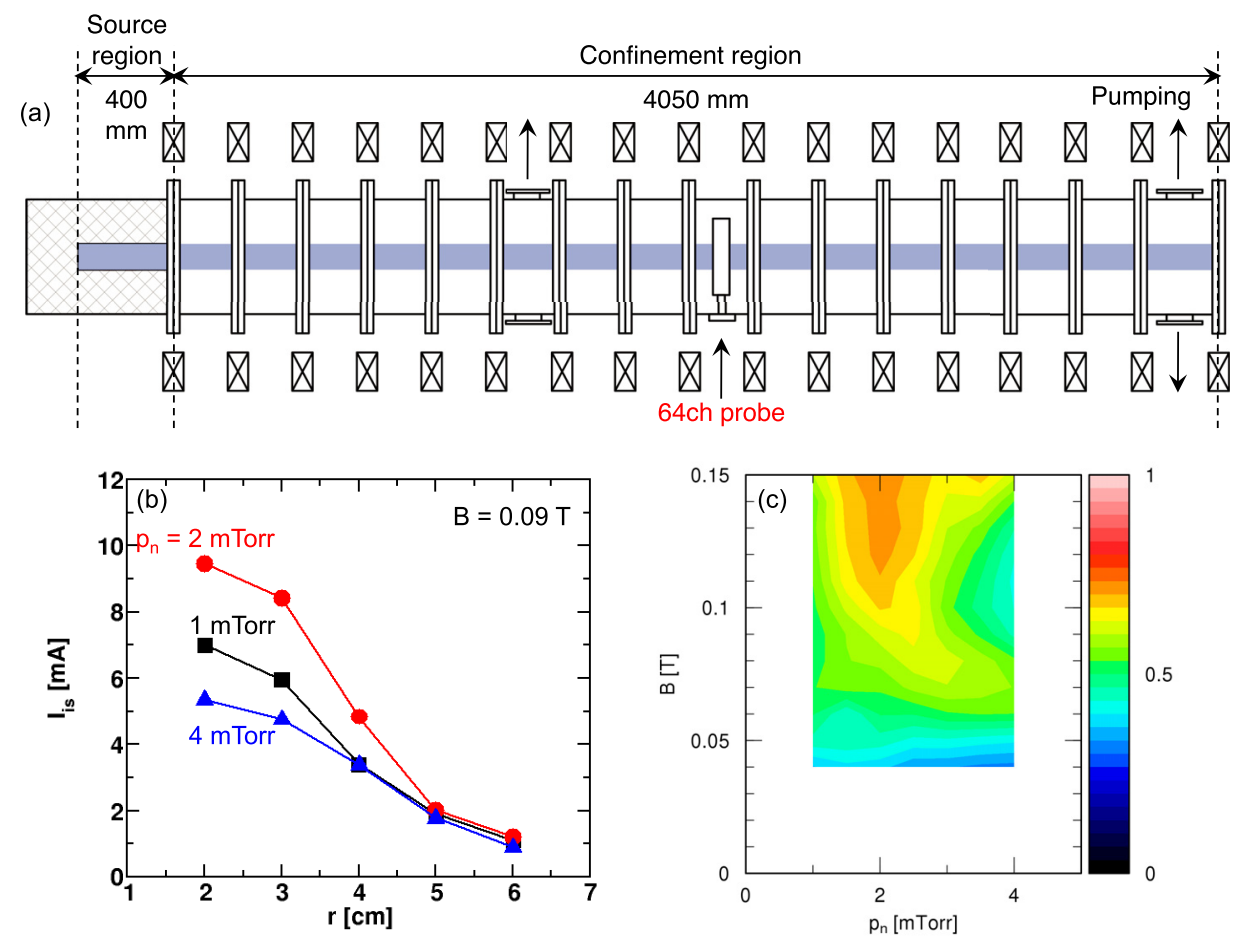

Fig. 1 (a) Schematic view of PANTA, (b) Radial profiles of the mean ion saturation current measured at $B=0.09 \mathrm{~T}$ and $p_{\mathrm{n}}=1,2$ and 4 mTorr conditions, (c) $p_{\mathrm{n}}-B$ map of the inverse gradient length of the mean ion saturation current in the unit of $\mathrm{cm}^{-1}$ at $r=4 \mathrm{~cm}$. 
Table 1 Typical operation conditions in the PANTA.

\begin{tabular}{c|c|c}
\hline Parameters & Typical value & Range \\
\hline \hline Filling gas pressure & $0.11 \mathrm{~Pa}(0.8 \mathrm{mTorr})$ & $0.11-0.63 \mathrm{~Pa}(0.8-4.8 \mathrm{mTorr})$ \\
Magnetic field & $0.09 \mathrm{~T}$ & $0.04-0.15 \mathrm{~T}$ \\
RF power & $3 \mathrm{~kW}$ & $0.5-5 \mathrm{~kW}$ \\
Electron density at center & $6 \times 10^{18} \mathrm{~m}^{-3}$ & $10^{18}-10^{19} \mathrm{~m}^{-3}$ \\
Electron temperature at center & $3 \mathrm{eV}$ & $1-4 \mathrm{eV}$ \\
\hline
\end{tabular}
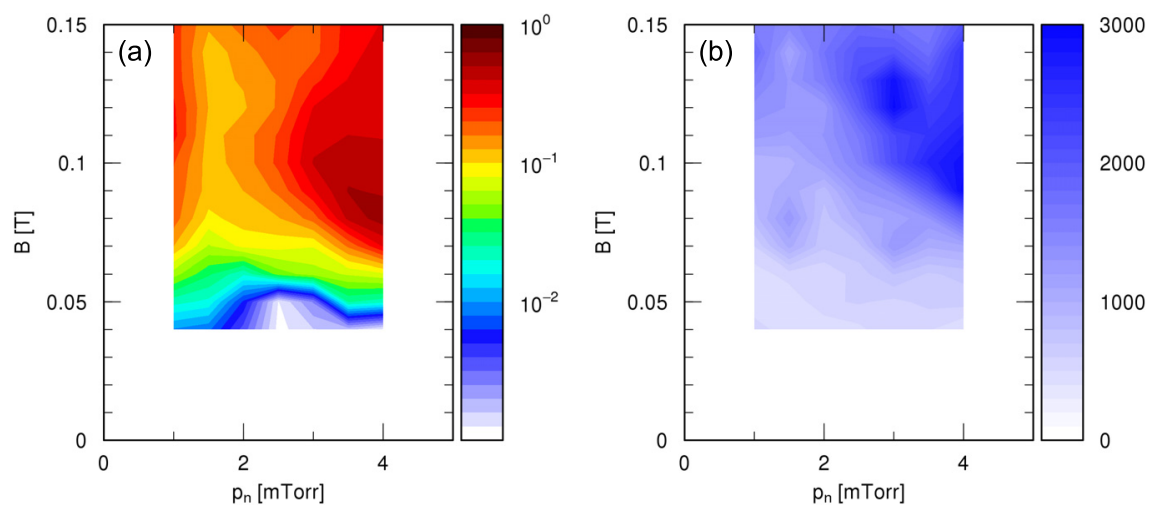

Fig. $2 p_{\mathrm{n}}-B$ maps of the (a) total fluctuation power and (b) total squared bicoherence at $r=3-5 \mathrm{~cm}$.

electrostatic probe arrays. Azimuthal turbulence structure is measured with a 64-channel probe array [27] installed at $z=2.125 \mathrm{~m}$, where $z$ is defined as the axial distance from the edge of the vacuum chamber at the source region side. The 64-channel probe tips made of tungsten wire with a diameter of $\phi=0.8 \mathrm{~cm}$ and a length of $l=3 \mathrm{~mm}$ is aligned annularly at a radius of $r=4 \mathrm{~cm}$. The probe tips measure the ion saturation current and the floating potential alternately, although only the ion saturation current data is analyzed in the article. The radial profile of the ion saturation current is measured with a 5-channel radial rake probe installed at $z=1.625 \mathrm{~m}$ [28]. The radial rake probe covers from $r=2 \mathrm{~cm}$ to $r=6 \mathrm{~cm}$ with a spacing of $\Delta r=1 \mathrm{~cm}$. The electron temperature fluctuation is considered to be small so that the normalized ion saturation current fluctuation is regarded as the normalized density fluctuation, $\tilde{I}_{\text {is }} / \bar{I}_{\text {is }} \sim \tilde{n}_{\mathrm{e}} / \bar{n}_{\mathrm{e}}$. Data analysis is performed for the time period of $\Delta t=290 \mathrm{~ms}$ out of the whole discharge of $500 \mathrm{~ms}$, in which the turbulent spectrum is quasi-saturated. That time period is much longer than the typical turbulence time period of $\leq 1 \mathrm{~ms}$.

\section{Fundamental Characteristics of Streamer State and Solitary Wave State}

In this section, the results of the systematic $B-p_{\mathrm{n}}$ scan experiments are shown. Characteristic features of the streamer state and the solitary wave state are exhibited based on spatiotemporal evolution of the ion saturation current and its power spectral density.

\subsection{Ion saturation current profile and fluctu- ation amplitude}

Figure 1 (b) shows typical radial profiles of the ion saturation current at $B=0.09 \mathrm{~T}$ and $p_{\mathrm{n}}=1,2$, and 4 mTorr, respectively. The central ion saturation current does not increase monotonically, as the neutral pressure is increased. This may be because the plasma source and the ion-neutral collision frequency are simultaneously altered as $p_{\mathrm{n}}$ is altered. At high $p_{\mathrm{n}}$, an increased density gradient sustained by a higher plasma source input contributes to a stronger linear drift wave excitation. At the same time the excited drift wave is subject to a stronger ion-neutral collisional damping in high $p_{\mathrm{n}}$ condition [7,8]. Figure 1 (c) plots the inverse gradient length of the ion saturation current $L=-I_{\text {is }}^{-1} \partial I_{\text {is }} / \partial r$ in the unit of $\mathrm{cm}^{-1}$ as a function of $B$ and $p_{\mathrm{n}}$ at $r=4 \mathrm{~cm}$. The complicated dependence of the inverse gradient length of the ion saturation current on the control parameters might be explained by the nonlinear plasma turbulence structural formation.

Figure 2 (a) indicates the $B-p_{\mathrm{n}}$ dependence of the integrated fluctuation power of the ion saturation current defined as

$$
S_{\mathrm{T}}^{2}=\int_{3 \mathrm{~cm}}^{5 \mathrm{~cm}} \mathrm{~d} r \int_{\Delta f}^{F} \mathrm{~d} f S^{2}(r, f),
$$

where $S^{2}(r, f)$ is the Fourier power spectral density of the ion saturation current fluctuation. In this article, Fourier transform (FT) is performed with the time window of $T=5 \mathrm{~ms}$ multiplied by the Tukey window function. The frequency resolution is therefore $\Delta f=T^{-1}=200 \mathrm{~Hz}$. The upper limit frequency of interest is taken to be $F=50 \mathrm{kHz}$, 
above which the fluctuation amplitude is negligibly small. The relative ion saturation current fluctuation power is integrated from $3 \mathrm{~cm}$ to $5 \mathrm{~cm}$, around which the strongest gradient appears. Two peaks in the $B-p_{\mathrm{n}}$ map of the power spectrum can be seen at low $p_{\mathrm{n}}$ and intermediate to high $B$ region and high $p_{\mathrm{n}}$ and intermediate $B$ region. The strong fluctuation power looks corresponding to the smaller gradient as shown in Fig. 1 (c), except for the low $B$ region. Shown in Fig. 2 (b) is the $B-p_{\mathrm{n}}$ map of the total squared auto bicoherence defined as,

$$
b_{\mathrm{T}}^{2}=\int_{3 \mathrm{~cm}}^{5 \mathrm{~cm}} \mathrm{~d} r \int_{\Delta f}^{F} \mathrm{~d} f^{\prime \prime} \int_{f^{\prime}}^{F} \mathrm{~d} f b^{2}\left(r, f, f^{\prime}\right),
$$

where $b^{2}\left(r, f, f^{\prime}\right)$ is the auto bicoherence of the ion satu- ration current fluctuation in which the matching condition $f^{\prime \prime}=f \pm f^{\prime}$ is satisfied $[6,24,29]$. The higher total squared bicoherence at high $p_{\mathrm{n}}$ and intermediate $B$ region corresponds to the peak in the fluctuation power.

\subsection{Observed two turbulence states; streamer state and solitary wave state}

As mentioned in introduction, nonlinear turbulent structures in PANTA can be classified into two different states, i.e., the streamer state and the solitary wave state. Here, characteristics of these two turbulence states are discussed in detail. Figure 3 shows the typical spatiotemporal evolutions plotted in time - azimuthal angle space. The positive direction of the azimuthal angle is taken as the
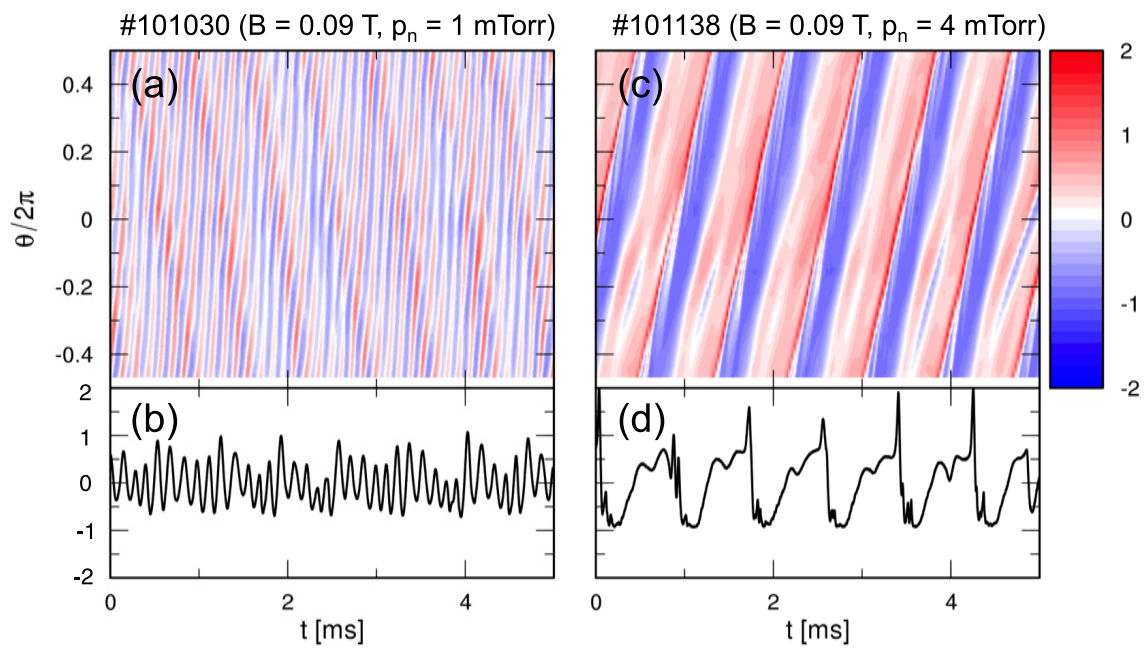

Fig. 3 (left column) (a) Spatiotemporal evolution of the relative ion saturation current fluctuation at $B=0.09 \mathrm{~T}$ and $p_{\mathrm{n}}=1 \mathrm{mTorr}$ condition and (b) its spatial slices at $\theta / 2 \pi=0$. (right column) (c) and (d) those for $B=0.09 \mathrm{~T}$ and $p_{\mathrm{n}}=4$ mTorr condition.
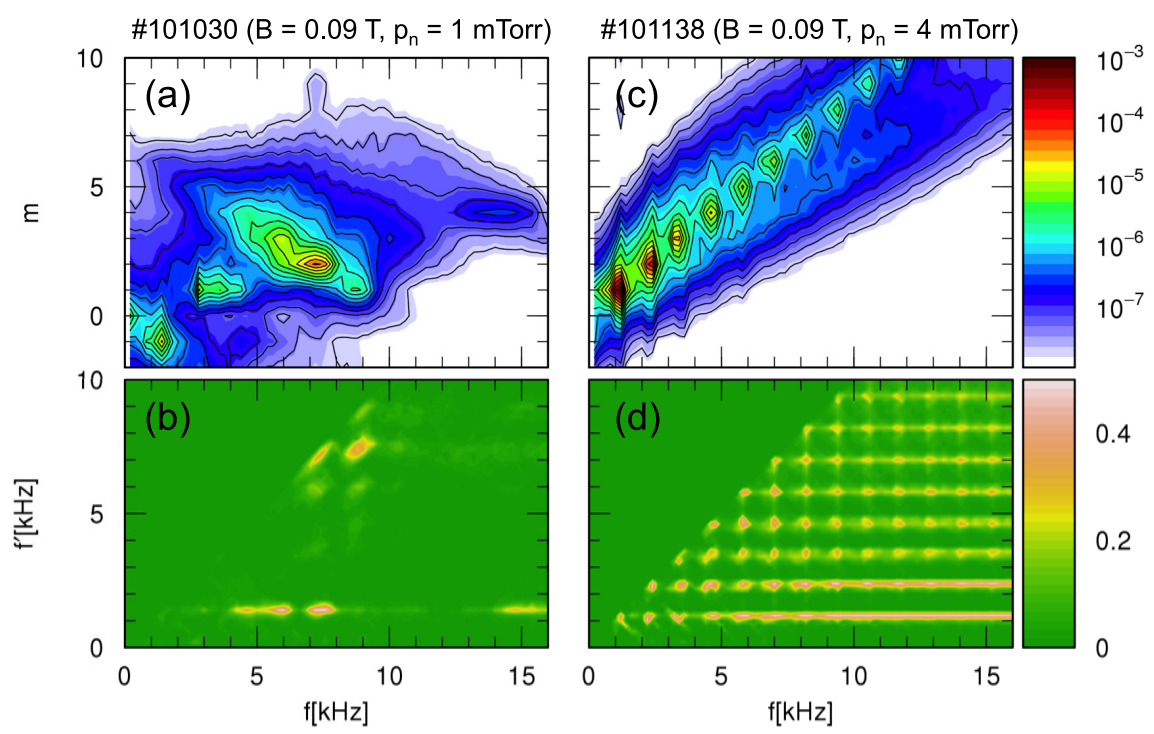

Fig. 4 (left column) (a) Frequency $f$ and azimuthal mode number $m$ spectra (dispersion relation) and (b) local squared bicoherence at $\theta / 2 \pi=0$ of the relative ion saturation current fluctuation at $B=0.09 \mathrm{~T}$ and $p_{\mathrm{n}}=1 \mathrm{mTorr}$ condition. (right column) (c) and (d) those for $B=0.09 \mathrm{~T}$ and $p_{\mathrm{n}}=4 \mathrm{mTorr}$ condition. 
right hand rule of the axial magnetic field direction, i.e., the electron diamagnetic drift direction. By keeping $B$ to be an intermediate value of $0.09 \mathrm{~T}$ and increasing $p_{\mathrm{n}}$, the structural transition from the streamer state to the solitary wave state can be observed.

In the discharge with low $p_{\mathrm{n}}$ and intermediate $B$, here $\left(p_{\mathrm{n}}, B\right)=(1 \mathrm{mTorr}, 0.09 \mathrm{~T})$, a typical streamer structure is excited as shown in Fig. 3 (a). The primary linear instability is identified to be the drift wave that propagates in the electron diamagnetic drift direction. The linearly unstable drift wave is nonlinearly bunched that forms a radially elongated convective cell [6] as predicted by an analytical study [11]. The envelope propagates in the opposite direction of the drift wave. Figure 4 (a) shows the frequency $f$-mode number $m$ decomposed power spectral density for the discharge shown in Fig. 3 (a). Two-dimensional FT $X(f, m)$ of a two-dimensional signal $x(t, \theta)$ is defined as,

$$
\begin{aligned}
& X(f, m) \\
& \quad=\frac{1}{T} \int_{0}^{T} \mathrm{~d} t \int_{0}^{2 \pi} \mathrm{d} \theta x(t, \theta) \exp [i(2 \pi f t-m \theta)],
\end{aligned}
$$

where $i$ is the imaginary unit. The two-dimensional power spectral density is calculated as $|X(f, m)|^{2} / \Delta f$. The dominant drift wave has a broadband spectrum at $f \sim 7 \mathrm{kHz}$ and $m=2$. A low frequency mode propagating in the opposite direction is also observed at $f \sim 1.4 \mathrm{kHz}$ and $m=-1$, which is the key for forming the streamer structure the so-called mediator. The mediator is considered to be excited nonlinearly by the drift waves, and to be responsible for the further broadening of the drift wave spectrum. The nonlinear broadening of the spectrum results in the nonlinear bunching seen in the spatiotemporal evolution. Figure 4(b) shows the squared auto bicoherence showing the evidence of that nonlinear coupling. Bicoherence takes a large value at the region $4 \mathrm{kHz} \leq f \leq 8 \mathrm{kHz}$ and $f^{\prime} \sim 1.4 \mathrm{kHz}$, which shows the strong nonlinear coupling between the drift waves and the mediator. Peaks around $7 \mathrm{kHz} \leq f \leq 9 \mathrm{kHz}$ and $7 \mathrm{kHz} \leq f^{\prime} \leq 8 \mathrm{kHz}$ corresponds to the self coupling of the drift waves that generates the higher harmonics.

Increasing $p_{\mathrm{n}}$ up to 4.0 mTorr exhibits the transition from the streamer state to the solitary wave state. Figure 3 (c) indicates the spatiotemporal evolution of the relative ion saturation current fluctuation when the solitary wave is excited. A sawtooth wave-like structure having a large amplitude propagates in the electron diamagnetic drift direction with a constant speed, which is relatively slower than the propagation speed of the drift wave seen in the streamer state. The waveform looks strongly distorted, creating an week gradient in the front and a strong gradient in the back. The frequency $f$-mode number $m$ decomposed power spectral density for the case of the solitary wave state is shown in Fig. 4(c). There are a distinguishable dominant mode at $f \sim 1.2 \mathrm{kHz}$ and $m=1$ and its higher harmonics. All these modes have an almost identical propagation velocity in the laboratory frame, that maintains the strongly distorted waveform propagating as a solitary wave. Figure 4 (d) shows the squared auto bicoherence for the case of the solitary wave. There are the regularly aligned peaks on a lattice pattern. This shows that the strong mutual nonlinear couplings among the excited modes exist, forming a nonlinear phase locking system as predicted in Ref. [18].

\section{Strength of Streamer}

As discussed on Fig. 3 (a), the characteristic feature of the streamer state is the envelope of the drift wave propagating in the ion diamagnetic drift direction. The prerequisite conditions are that (1) the mediator propagating in the ion diamagnetic drift direction exists and (2) the envelope of the drift wave is modulated by the mediator. Therefore, here we define the strength of the streamer as two values; the total fluctuation power of the modes propagating in the ion diamagnetic drift direction and the coherence between the envelope of the modes propagating in the electron diamagnetic drift direction and the modes propagating in the ion diamagnetic drift direction. Note that a streamer having the envelope propagating in the electron diamagnetic drift direction is also possible in principle $[7,8]$, but in the experiment these streamers have not yet been observed in the present parameter regime.

The above two criterions are evaluated by means of the filter technique using the two-dimensional FT and the inverse FT (IFT). The time evolution of the fluctuation waveform that has a specific mode number $m_{1} \leq m \leq m_{2}$ can be reconstructed by the partial IFT defined as,

$$
\hat{x}_{m_{1} \leq m \leq m_{2}}(t)=\int_{m_{1}}^{m_{2}} \mathrm{~d} m \int_{\Delta f}^{F} \mathrm{~d} f X(f, m) \exp [-i 2 \pi f t],
$$

where $X(f, m)$ is the two-dimensional Fourier spectrum of a signal of interest. The ion diamagnetic directed mode $\hat{x}_{\mathrm{i}}$ and the electron diamagnetic directed mode $\hat{x}_{\mathrm{e}}$ are defined as,

$$
\hat{x}_{\mathrm{i}}(t)=\hat{x}_{-4 \leq m \leq-1}(t),
$$

and

$$
\hat{x}_{\mathrm{e}}(t)=\hat{x}_{1 \leq m \leq 6}(t),
$$

respectively. The envelope of the electron diamagnetic directed mode is calculated as

$$
\hat{E}_{\mathrm{e}}(t)=\sqrt{\hat{x}_{\mathrm{e}}^{2}(t)+\left(H\left[\hat{x}_{\mathrm{e}}(t)\right]\right)^{2}}
$$

where $H[\cdot]$ is Hilbert transform for a time series. An application of the envelope analysis using Hilbert transform for investigating the streamer can be seen in Ref. [30]. In order to quantify the strength of the streamer, the power spectrum of $\hat{x}_{\mathrm{i}}(t)$ and the squared cross coherence between $\hat{x}_{\mathrm{i}}(t)$ and $\hat{E}_{\mathrm{e}}(t)$, which are denoted as $S_{\hat{x}_{\mathrm{i}}}^{2}(f)$ and $\gamma_{\hat{x}_{\mathrm{i}}, \hat{E}_{\mathrm{e}}}^{2}(f)$ respectively, are calculated. Then, the frequency domain 

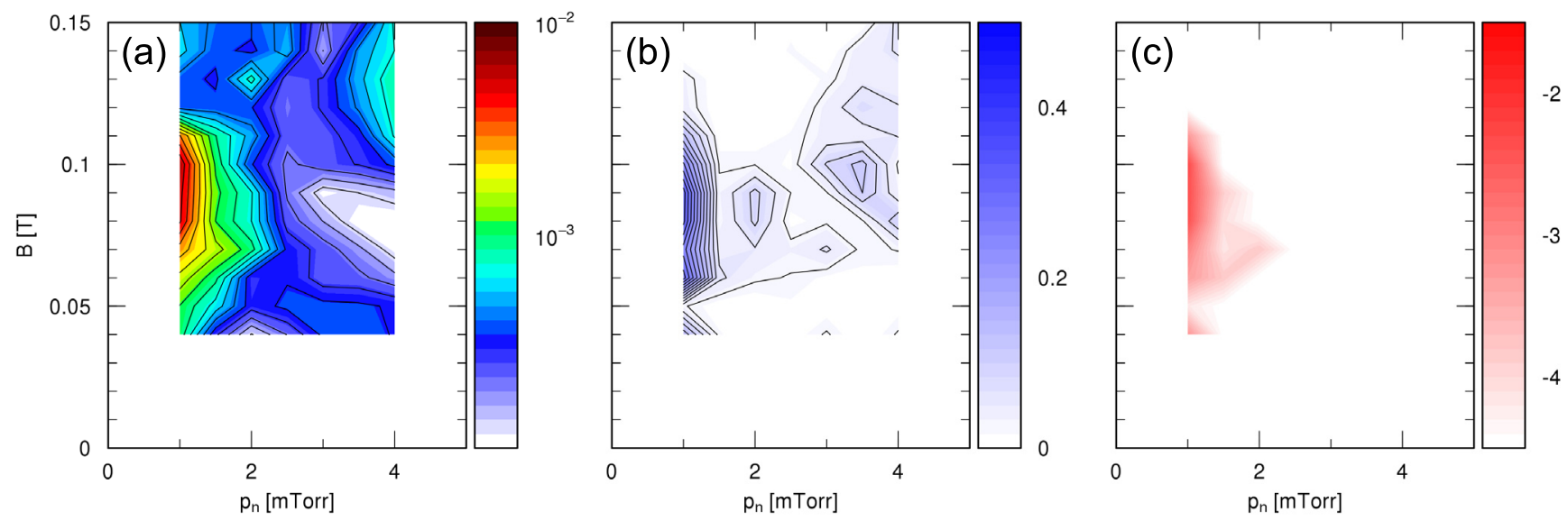

Fig. $5 p_{\mathrm{n}}-B$ maps of (a) the integrated power spectrum of the ion diamagnetic propagation directed mode, (b) the integrated squared cross coherence between $\hat{x}_{\mathrm{i}}(t)$ and $\hat{E}_{\mathrm{e}}(t)$ in the arbitrary unit, and (c) the streamer strength $I_{\mathrm{S}}$.

integration is performed in the range of $\left[f_{\mathrm{p}}-\delta f_{\mathrm{p}}, f_{\mathrm{p}}+\delta f_{\mathrm{p}}\right]$, where $f_{\mathrm{p}}$ and $\delta f_{\mathrm{p}}$ denote the spectral peak frequency and the half frequency width at half maximum of $S_{\hat{x}_{\mathrm{i}}}^{2}(f)$. Figures 5 (a) and (b) show the integrated $S_{\hat{x}_{\mathrm{i}}}^{2}$ and $\gamma_{\hat{x}_{\mathrm{i}}, \hat{E}_{\mathrm{e}}}^{2}$, respectively. We define the streamer strength as

$$
I_{\mathrm{S}}=\log _{10}\left(\int_{f_{\mathrm{p}}-\delta f_{\mathrm{p}}}^{f_{\mathrm{p}}+\delta f_{\mathrm{p}}} \mathrm{d} f S_{\hat{x}_{\mathrm{i}}}^{2} \gamma_{\hat{x}_{\mathrm{i}}, \hat{E}_{\mathrm{e}}}^{2}\right),
$$

as shown in Fig. 5 (c). When the streamer strength is less than a threshold value of -4.5 , which corresponds to the lower limit of the contour plot, clear modulation pattern as shown in Fig. 3 (a) disappears.

\section{Strength of Solitary Wave}

In the solitary wave system, all the modes have a same phase velocity. To quantify this characteristic, we define the power weighted phase velocity correlation. The phase velocity on the plasma frame is proportional to the ratio between the mode frequency $f$ and the drift wave frequency $f_{*} \equiv v_{\mathrm{de}} m / r$, where $v_{\mathrm{de}}=T_{\mathrm{e}} / e B\left(-n_{\mathrm{e}}^{\prime} / n_{\mathrm{e}}\right)$ is the electron diamagnetic drift velocity. The two-dimensional Fourier component $X(f, m)$ can be written as $X\left(f, f_{*}\right)$. The power weighted phase velocity correlation is defined as

$$
\gamma_{\mathrm{ph}}^{2}=\frac{\left\langle\left|X\left(f, f_{*}\right)\right|^{2} \exp [i \alpha]\right\rangle}{\left\langle\left|X\left(f, f_{*}\right)\right|^{2}\right\rangle},
$$

where $\tan \alpha \equiv f_{*} / f$ is proportional to the phase velocity. The angle bracket $\langle\cdot\rangle$ indicates averaging in the regime $\Delta f<f<F$ and $-M<m<M$, where $M$ indicates the Nyquist mode number of the measurement system. The value $\gamma_{\mathrm{ph}}^{2}$ takes the maximal value of the unity when all the modes have an identical phase velocity, while existence of the broadband mode lessens $\gamma_{\mathrm{ph}}^{2}$. Figure 6 shows the power weighted phase velocity correlation as a function of $B$ and $p_{\mathrm{n}}$. The lower limit of the contour color map in Fig. 6 of $\gamma_{\mathrm{ph}}^{2}=0.95$ corresponds to an empirical threshold value for the solitary wave strength. In the region having $\gamma_{\mathrm{ph}}^{2}<0.95$,

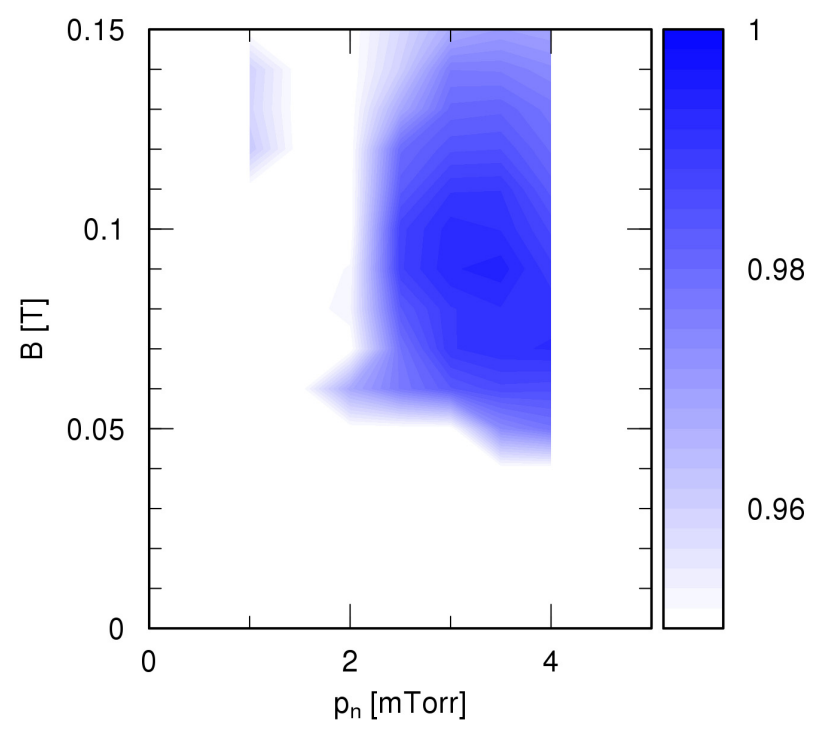

Fig. $6 p_{\mathrm{n}}-B$ map of the power weighted phase velocity correlation $\gamma_{\mathrm{ph}}^{2}$.

broadening of the spectrum is seen and the solitary wave nature is weaken. With intermediate $B$ and higher $p_{\mathrm{n}}$ values $\gamma_{\mathrm{ph}}^{2}$ takes a larger value, showing that the spectral shape is solitary wave like.

\section{Discussion and Summary}

By superposing Fig. 5 (c) and Fig. 6, occurrence regions of the streamer state and the solitary wave state, which we call the phenomenological classification diagram, is depicted in Fig. 7. In the region where the magnetic field is low $(B<0.06 \mathrm{~T})$, the linear unstable drift wave amplitude is small so that neither the streamer nor the solitary wave is formed, except for the discharge with the low neutral pressure. In the region having a intermediate magnetic field and a intermediate neutral pressure, the occurrence regions of the two states approach each other. In- 


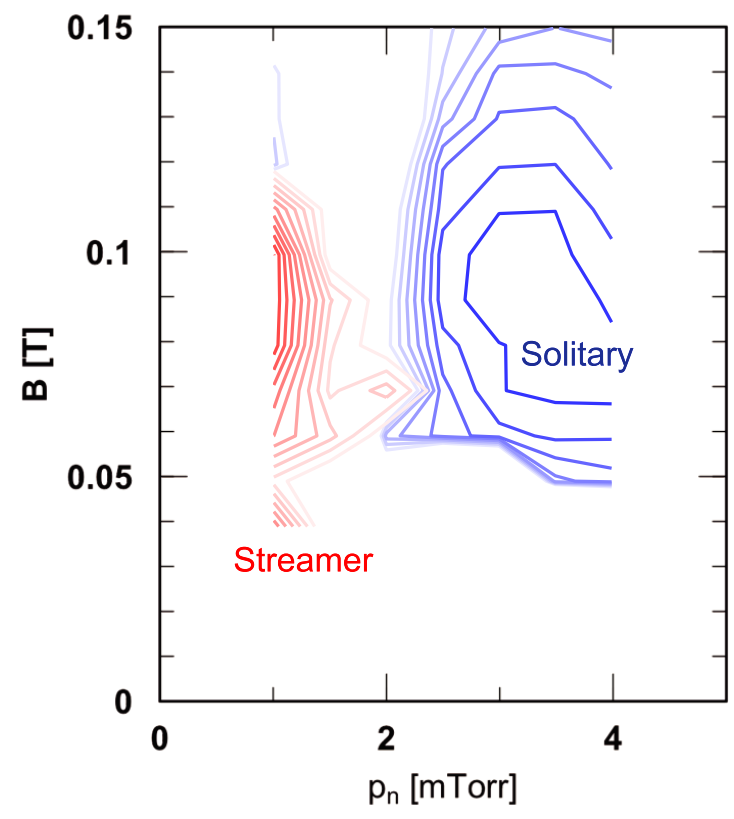

Fig. $7 p_{\mathrm{n}}-B$ map of the phenomenological classification diagram (see the text for details).

terestingly, an abrupt and spontaneous transition between the two nonlinear turbulence states occurs in this parameter regime [21,22]. The existence of the transition region at which the two regions are adjacent may imply that the present choice of the threshold values is valid. When the magnetic field is high and the neutral pressure is low, turbulence state is classified neither in the streamer state nor in the solitary wave state. The regime where these two nonlinear states emerge corresponds to the regimes where the inverse gradient length of the ion saturation current profile is small [shown in Fig. 1 (c)] and the mode fluctuation power is large [shown in Fig. 2 (a)]. The total bicoherence is particularly large in the solitary wave state [shown in Fig. 2 (b)].

In this paper, we performed a parameter scan experiments in the magnetic field strength and the neutral pressure parameter space. During the parameter scan, two different nonlinear turbulent states, i.e., the streamer state and the solitary wave state, were observed. We defined the strength of the streamer and the solitary wave phenomenologically, by which the occurrence region of these two states were determined.

\section{Acknowledgments}

We thank profs. K. Ida and S. Sakakibara for strong support. This work is partly supported by the Grant-in-Aid for Scientific Research of JSPS (15H02155), the collaboration programs of RIAM of Kyushu University and NIFS, and Asada Science Foundation.

[1] U. Stroth, J. Adamek, L. Aho-Mantila, S. Äkäslompolo, C. Amdor, C. Angioni, M. Balden, S. Bardin, L. Barrera Orte,
K. Behler et al., Nucl. Fusion 53, 104003 (2013).

[2] C.F. Maggi, E. Delabie, T.M. Biewer, M. Groth, N.C. Hawkes, M. Lehnen, E. de la Luna, K. McCormick, C. Reux, F. Rimini et al., Nucl. Fusion 54, 023007 (2014).

[3] T.N. Carlstrom and R.J. Groebner, Phys. Plasmas 3, 1867 (1996).

[4] M.J. Burin, G.R. Tynan, G.Y. Antar, N.A. Crocker and C. Holland, Phys. Plasmas 12, 052320 (2005).

[5] F. Brochard, E. Gravier and G. Bonhomme, Phys. Plasmas 12, 062104 (2005).

[6] T. Yamada, S.-I. Itoh, S. Inagaki, Y. Nagashima, N. Kasuya, K. Kamataki, H. Arakawa, T. Kobayashi, M. Yagi, A. Fujisawa et al., Phys. Rev. Lett. 105, 225002 (2010).

[7] N. Kasuya, M. Yagi, K. Itoh and S.-I. Itoh, Phys. Plasmas 15, 052302 (2008).

[8] M. Sasaki, N. Kasuya, K. Itoh, M. Yagi and S.-I. Itoh, Nucl. Fusion 54, 114009 (2014).

[9] P.A. Politzer, Phys. Rev. Lett. 84, 1192 (2000).

[10] P. Beyer, S. Benkadda, X. Garbet and P.H. Diamond, Phys. Rev. Lett. 85, 4892 (2000).

[11] K. Nozaki, T. Taniuti and K. Watanabe, J. Phys. Soc. Jpn. 46, 991 (1979).

[12] T. Yamada, S.-I. Itoh, T. Maruta, N. Kasuya, Y. Nagashima, S. Shinohara, K. Terasaka, M. Yagi, S. Inagaki, Y. Kawai et al., Nat. Phys. 4, 721 (2008).

[13] S. Inagaki, T. Kobayashi, Y. Kosuga, S.-I. Itoh, T. Mitsuzono, Y. Nagashima, H. Arakawa, T. Yamada, Y. Miwa, N. Kasuya et al., Sci. Rep. 6, 22189 (2016).

[14] T. Kobayashi, S. Inagaki, Y. Kosuga, M. Sasaki, Y. Nagashima, T. Yamada, H. Arakawa, N. Kasuya, A. Fujisawa, S.-I. Itoh and K. Itoh, Phys. Plasmas 23, 102311 (2016).

[15] H. Arakawa, T. Kobayashi, S. Inagaki, N. Kasuya, S. Oldenbürger, Y. Nagashima, T. Yamada, M. Yagi, A. Fujisawa, K. Itoh and S.-I. Itoh, Plasma Phys. Control. Fusion 53, 115009 (2011).

[16] T. Kobayashi, S. Inagaki, H. Arakawa, S. Oldenbüeger, M. Sasaki, Y. Nagashima, T. Yamada, S. Sugita, M. Yagi, N. Kasuya, A. Fujisawa, S.-I. Itoh and K. Itoh, Plasma Fusion Res. 6, 2401082 (2011).

[17] T. Kobayashi, S. Inagaki, M. Sasaki, Y. Kosuga, H. Arakawa, T. Yamada, Y. Nagashima, Y. Miwa, N. Kasuya, A. Fujisawa et al., Phys. Plasmas 22, 112301 (2015).

[18] M. Sasaki, N. Kasuya, T. Kobayashi, H. Arakawa, K. Itoh, K. Fukunaga, T. Yamada, M. Yagi and S.-I. Itoh, Phys. Plasmas 22, 032315 (2015).

[19] S. Oldenbürger, S. Inagaki, T. Kobayashi, H. Arakawa, N. Ohyama, K. Kawashima, Y. Tobimatsu, A. Fujisawa, K. Itoh and S.-I. Itoh, Plasma Phys. Control. Fusion 54, 055002 (2012).

[20] H. Arakawa, S. Inagaki, M. Sasaki, Y. Kosuga, T. Kobayashi, N. Kasuya, Y. Nagashima, T. Yamada, M. Lesur, A. Fujisawa et al., Sci. Rep. 6, 33371 (2016).

[21] H. Arakawa, K. Kamataki, S. Inagaki, T. Maruta, Y. Nagashima, T. Yamada, S. Shinohara, K. Terasaka, S. Sugita, M. Yagi et al., Plasma Phys. Control. Fusion 51, 085001 (2009).

[22] H. Arakawa, S. Inagaki, Y. Nagashima, T. Yamada, K. Kamataki, T. Kobayashi, S. Sugita, M. Yagi, N. Kasuya, A. Fujisawa et al., Plasma Phys. Control. Fusion 52, 105009 (2010).

[23] K. Terasaka, S. Shinohara, Y. Nagashima, T. Yamada, M. Kawaguchi, T. Maruta, S. Inagaki, Y. Kawai, N. Kasuya, M. Yagi, A. Fujisawa, K. Itoh and S.-I. Itoh, Plasma Fusion 


\section{Res. 2, 31 (2007).}

[24] T. Yamada, S.-I. Itoh, T. Maruta, N. Kasuya, S. Shinohara, Y. Nagashima, K. Terasaka, S. Inagaki, Y. Kawai, M. Fukao et al., Plasma Fusion Res. 3, 044 (2008).

[25] S. Shinohara, T. Hada, T. Motomura, K. Tanaka, T. Tanikawa, K. Toki, Y. Tanaka and K.P. Shamrai, Phys. Plasmas 16, 057104 (2009).

[26] M. Ignatenko, M. Azumi, M. Yagi, S. Shinohara, S.-I. Itoh and K. Itoh, Jpn. J. Appl. Phys. 46, 1680 (2007).

[27] T. Yamada, Y. Nagashima, S. Inagaki, Y. Kawai, M. Yagi, S.-I. Itoh, T. Maruta, S. Shinohara, K. Terasaka, M.
Kawaguchi et al., Rev. Sci. Instrum. 78, 123501 (2007).

[28] N. Ohyama, A. Fujisawa, S. Inagaki, Y. Nagashima, S. Oldenbürger, T. Kobayashi, K. Kawashima, Y. Tobimatsu, T. Yamashita, S. Yamada et al., Plasma Fusion Res. 7, 1201025 (2012).

[29] Y.C. Kim and E.J. Powers, IEEE Trans. Plasma Sci. 7, 120 (1979).

[30] F. Kin, T. Yamada, S. Inagaki, H. Arakawa, Y. Nagashima, N. Kasuya, A. Fujisawa, K. Nakanishi, H. Kono, T. Mizokami et al., Plasma Fusion Res. 10, 3401043 (2015). 